\title{
Estimated Beam Loss due to Energy Straggling through the Charge Exchange Injection Foil
}

BNL/SNS TECHNICAL NOTE

NO. 058

D. Raparia

February 8, 1999

ALTERNATING GRADIENT SYNCHROTRON DEPARTMENT BROOKHAVEN NATIONAL LABORATORY UPTON, NEW YORK 11973 


\title{
Estimated Beam Loss due to Energy Straggling through the Charge Exchange Injection Foil
}

\author{
Deepak raparia
}

\section{Introduction}

As $\mathrm{H}^{-}$or circulating protons traverses the charge exchange injection foil, it deposits some of its energy in the foil. The energy distribution $\mathrm{f}(\Delta, \mathrm{s})$ depends on a parameter $\kappa$, which is proportional to the ratio of the mean energy loss over the path length to the largest energy transfer possible in a collision with an atomic electron. If $\kappa \rightarrow 0$, distribution is Landau [1], if $\kappa$ is between 1 and 10, then the distribution is Vavilov [2], and for $\kappa \geq 10$ then the distribution is Gaussian [2]. For the SNS accumulator ring injection foil, the parameter $\kappa$ is about $6.61 \times 10^{-6}$, and the largest energy transfer possible in a collision with an atomic electron is about 3.3 MeV. Therefore the distribution is Landau, with its tail extending up to $3.3 \mathrm{MeV}$. The concern was some of the protons, which lie in the tail of Landau distribution, will go through a large synchrotron oscillation and might end up the beam gap. These protons will be lost at time of extraction or at dispersive locations in the ring, and losses might be greater than or equal to $0.01 \%$.

\section{Significant Parameters}

The largest energy transfer possible in a collision between a particle of mass ' $M$ ' and a free electron of mass ' $m$ ' can be calculated by using the conservation of energy and momentum, and is given by

$\varepsilon_{\max }=\frac{2 m c^{2} \beta^{2} \gamma^{2}}{1+2 \gamma m / M+(m / M)^{2}}$

where $\beta$, and $\gamma$ are the relativistic parameters. For $1 \mathrm{GeV}$ proton $\varepsilon_{\max }$ is $3.3 \mathrm{MeV}$.

The mean energy loss in path-length $\mathrm{s}\left(\mathrm{g} \mathrm{cm}^{-2}\right)$ is given by the Bethe Bloch formula [3]

$$
\bar{\Delta}=0.30058 \frac{m c^{2}}{\beta^{2}} \frac{Z}{A} s\left[\log \left(\frac{2 m c^{2} \beta^{2} \varepsilon_{\max }}{I^{2}\left(1-\beta^{2}\right)}\right)-2 \beta^{2}-2 \frac{C}{Z}-\delta\right]
$$

Where $\mathrm{Z}, \mathrm{A}$, and I are the atomic mass number, atomic weight, and mean excitation energy of the medium, $2 \mathrm{C} / \mathrm{Z}$ is the shell correction, and $\delta$ is the density effect correction. For $200 \mu \mathrm{g} / \mathrm{cm}^{2}$ thick carbon foil average energy lost by $1 \mathrm{Gev}$ proton is $0.44 \mathrm{keV}$. 
The parameter $\kappa$ is defined as

$$
\begin{aligned}
& \kappa \equiv \frac{\xi}{\varepsilon_{\max }} \\
& \text { where } \xi=0.30058 \frac{m c^{2}}{\beta^{2}} \frac{Z}{A} s
\end{aligned}
$$

As $\kappa \rightarrow 0$ the distribution $\mathrm{f}(\Delta, \mathrm{s})$ is Landau, and if $\kappa \gg>1$ then the distribution $\mathrm{f}(\Delta, \mathrm{s})$ is Gaussian. For other values of $\kappa(=1-10)$ the distribution $f(\Delta, s)$ is Vavilov. For the SNS accumulator ring injection foil, the parameter $\kappa$ is about $6.61 \times 10^{-6}$.

The Landau parameter $\lambda$ is defined as

$\lambda=\frac{\Delta-\bar{\Delta}}{\xi}-1-\beta^{2}+C-\log \kappa$

Where $\Delta$ is the energy loss along the path length s and $\mathrm{C}=0.577216$ is Euler's constant.

The Landau distribution is

$f_{l}(\Delta, s)=\frac{1}{\xi} \phi(\lambda)$

where $\phi(\lambda)$ is universal function of the variable $\lambda$ only [2] and given by

$$
\phi(\lambda)=\frac{1}{\pi} \int_{0}^{\infty} e^{-\pi y / 2} \cos (y \log y+\lambda y) d y
$$

The Landau distribution $\mathrm{f}_{1}$ is asymmetric, with a tail extending to $\varepsilon_{\text {max }}$; it peaks at $\lambda=-0.233$ and has a full width at half maximum of $4.02 \lambda$.

\section{Method and Results}

To estimate proton beam losses the computer program ACCSIM [4] was used to simulate the injection and accumulation process. ACCSIM uses the CERN library subroutine DISLAN and DINLAN [5]. These subroutines cut off this distributions at $\lambda=-3.5$ and $\lambda=85.96782$. Figure 1 shows $\phi(\lambda)$ vs. $\lambda$, as given the subroutine DISLAN, and compared with equation 6. It has been shown by Borsch-Supan [6] that for large values of $\lambda$ the Landau distribution $\mathrm{f}(\lambda)=\mathrm{O}\left(1 / \lambda^{2}\right)$. Using this fact ACCSIM was modified to accommodate the full Landau tail. A test run was made for one billion $\left(10^{9}\right)$ foil traversals (foil thickness of $2000 \mu \mathrm{g} / \mathrm{cm}^{2}$, which is ten times thicker than the proposed SNS charge exchange foil). The resulting energy losses were binned and compared with equation $6,\left(\lambda_{\max } \sim 8300, \varepsilon_{\max }=3.3 \mathrm{MeV}\right.$ for a $1 \mathrm{GeV}$ proton $)$ and shown in figure 2.

To estimate particle loss ACCSIM runs (with modified subroutine) were made for $10^{5}$ particles. The first run was made with normal foil size $\left(8 \times 4.56 \mathrm{~mm}^{2}\right)$ and thickness $(200$ 
$\mu \mathrm{g} / \mathrm{cm}^{2}$ ). Figure 3 shows the ACCSIM output for $\mathrm{x}-\mathrm{x}^{\prime}, \mathrm{y}-\mathrm{y}, \mathrm{x}-\mathrm{y}$, and $\Delta \mathrm{E}-\phi$ phase space after 1225 turn. There was no particle loss and no particles in the beam gap. The average number of the foil traversals was four.

The second run was made with a full aperture foil $\left(100 \times 100 \mathrm{~mm}^{2}\right)$ which was ten times thicker $\left(2000 \mu \mathrm{g} / \mathrm{cm}^{2}\right)$. Figure 4 shows the ACCSIM output for $\mathrm{x}-\mathrm{x}, \mathrm{y}-\mathrm{y}, \mathrm{x}-\mathrm{y}$, and $\Delta \mathrm{E}-\phi$ phase space after 1225 turn. There were no particle losses but there were 302 particles in the beam gap. These 302 particles are considered as lost particles, as they will lost during the extraction. The average numbers of the foil traversals were 612 .

The ratio of average number of foil traversals of run 2 to run 1 is 153 , and foil factor due thickness is 10 . We therefore calculate the fractional beam loss due to energy straggling for a normal foil as:

Fractional Loss $=\frac{302}{10^{5} \times 10 \times 153}=2 \times 10^{-6}$

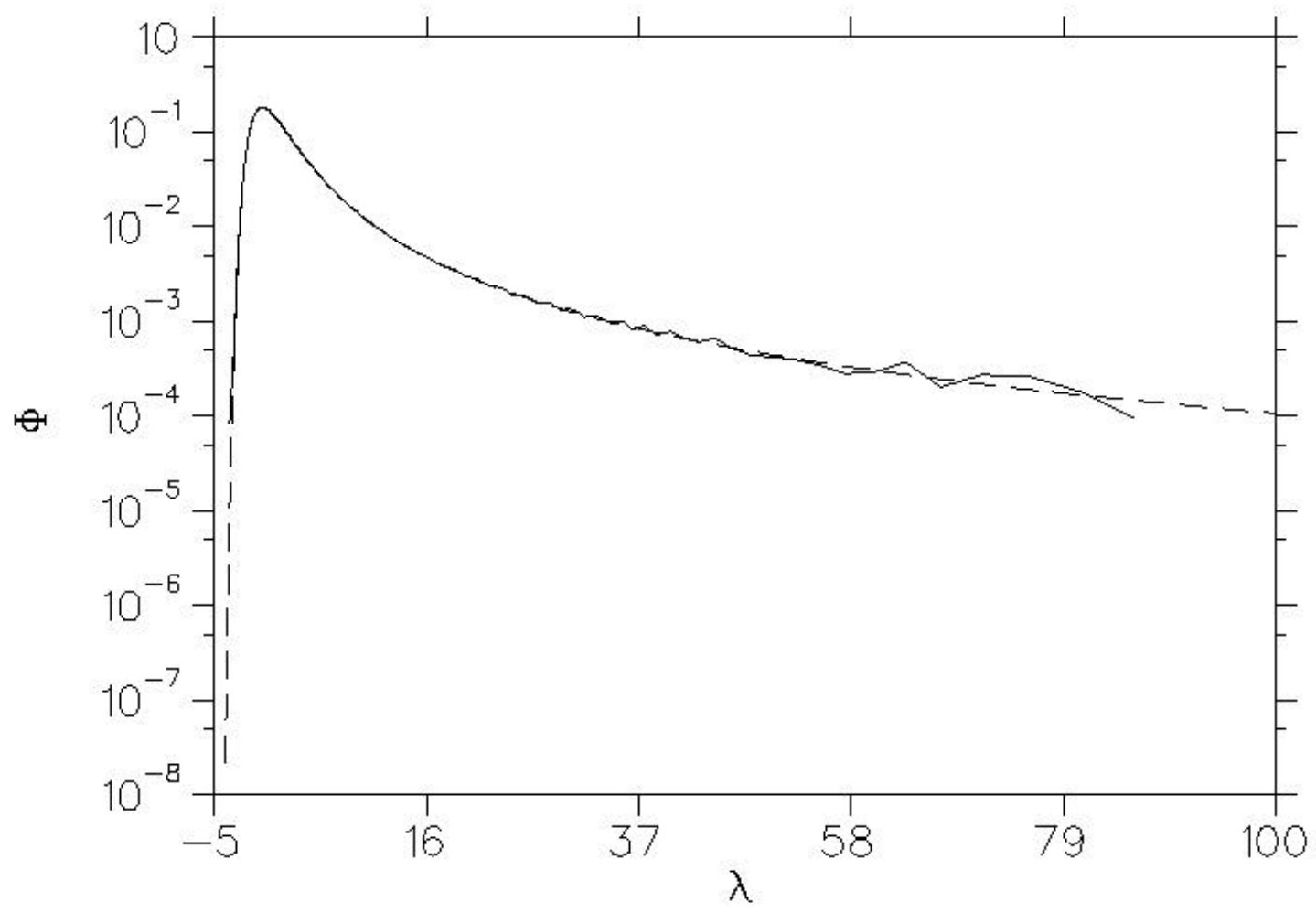

Figure 1: Comparison between the subroutine DISLAN (solid line) and Equation 6 (broken line) for the Landau distribution. 


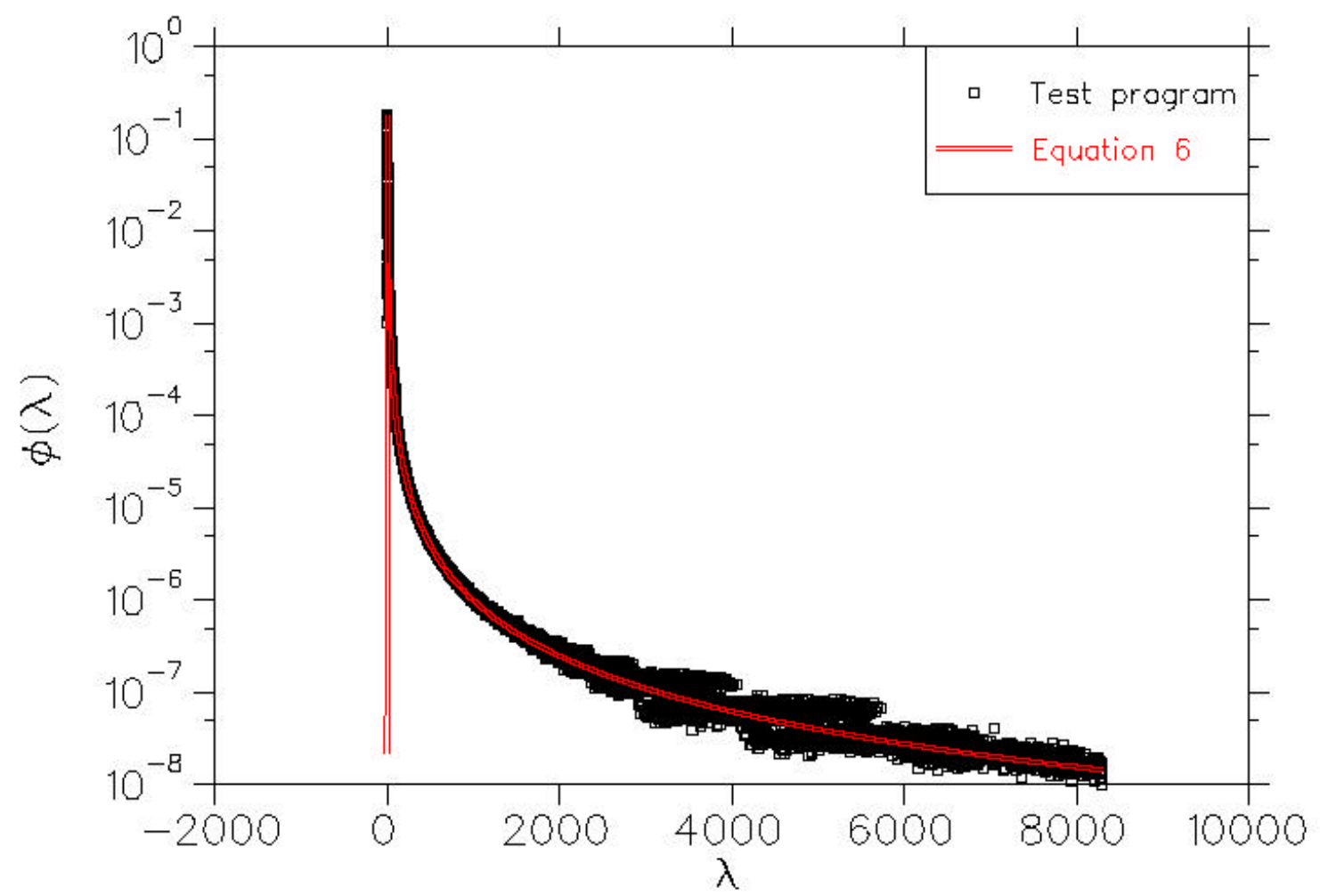

Figure 2: Comparison between the test program and Equation 6 for $10^{9}$ foil traversals (thickness $2000 \mu \mathrm{g} / \mathrm{cm}^{2}$ ). 

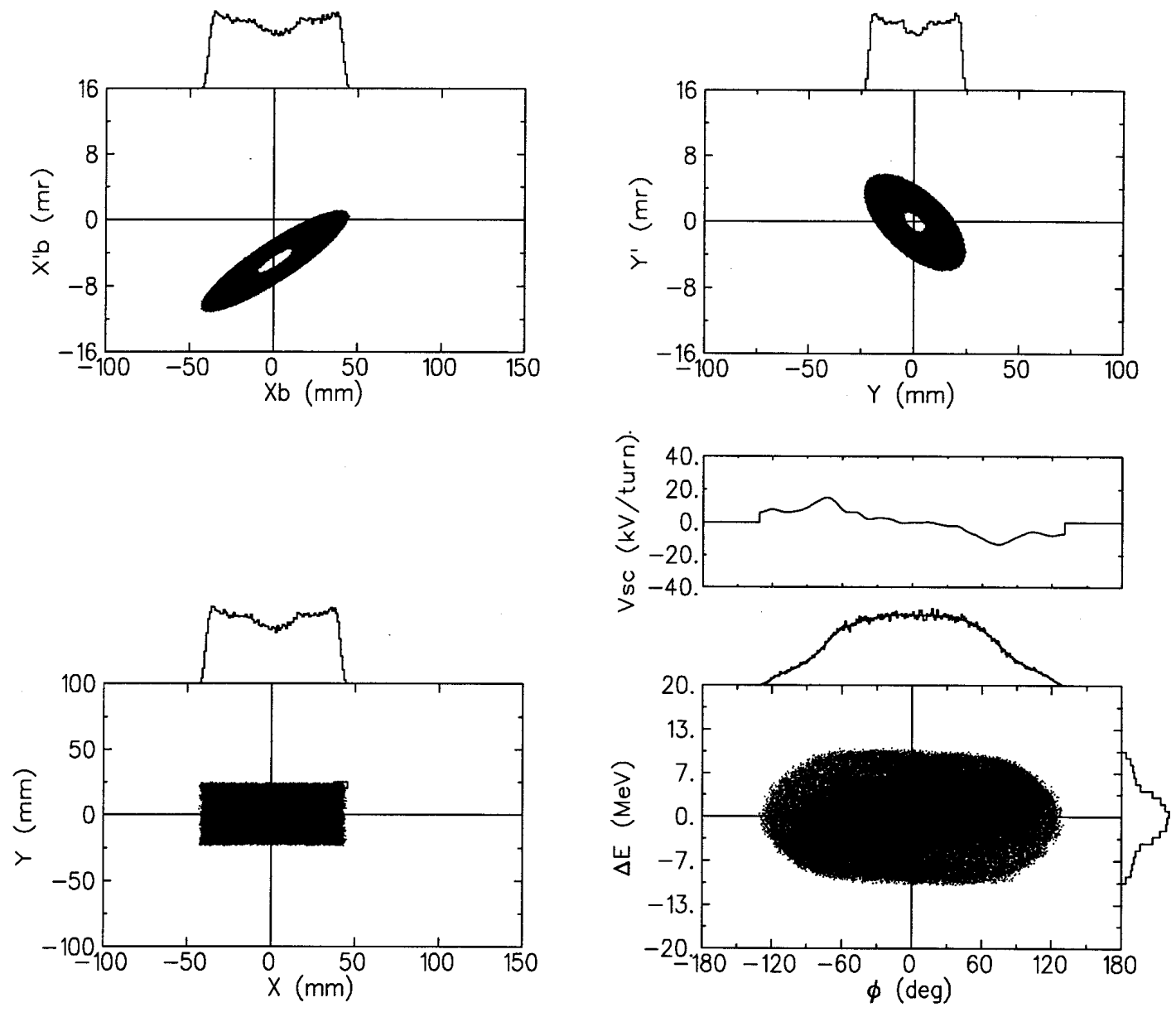

Turn \#, time (ms) $1225 \quad 1.03056$

At injection point

BETAx, BETAy $\quad 15.6400 \quad 5.17342$

ETAX, ETAxp 7.74552E-02 6.67709E-03

Ts, DEO (MeV) 1000.0000 .

Vrf (kV) 40.0000

CO bump (mm) $0.927327 \quad 0.530694$

Hits: total, average 3979664

Particles plotted, lost 979690

Real particles in circulating beam: $2.08334 \mathrm{E}+14$

sns01_D01

Dec 18 09:25:04 1998

ACCSIM v3.5j

Figure 3: ACCSIM output for $10^{5}$ particles with foil size $8 \times 4.58 \mathrm{~mm}^{2}$ and thickness 200 $\mu \mathrm{g} / \mathrm{cm}^{2}$. 

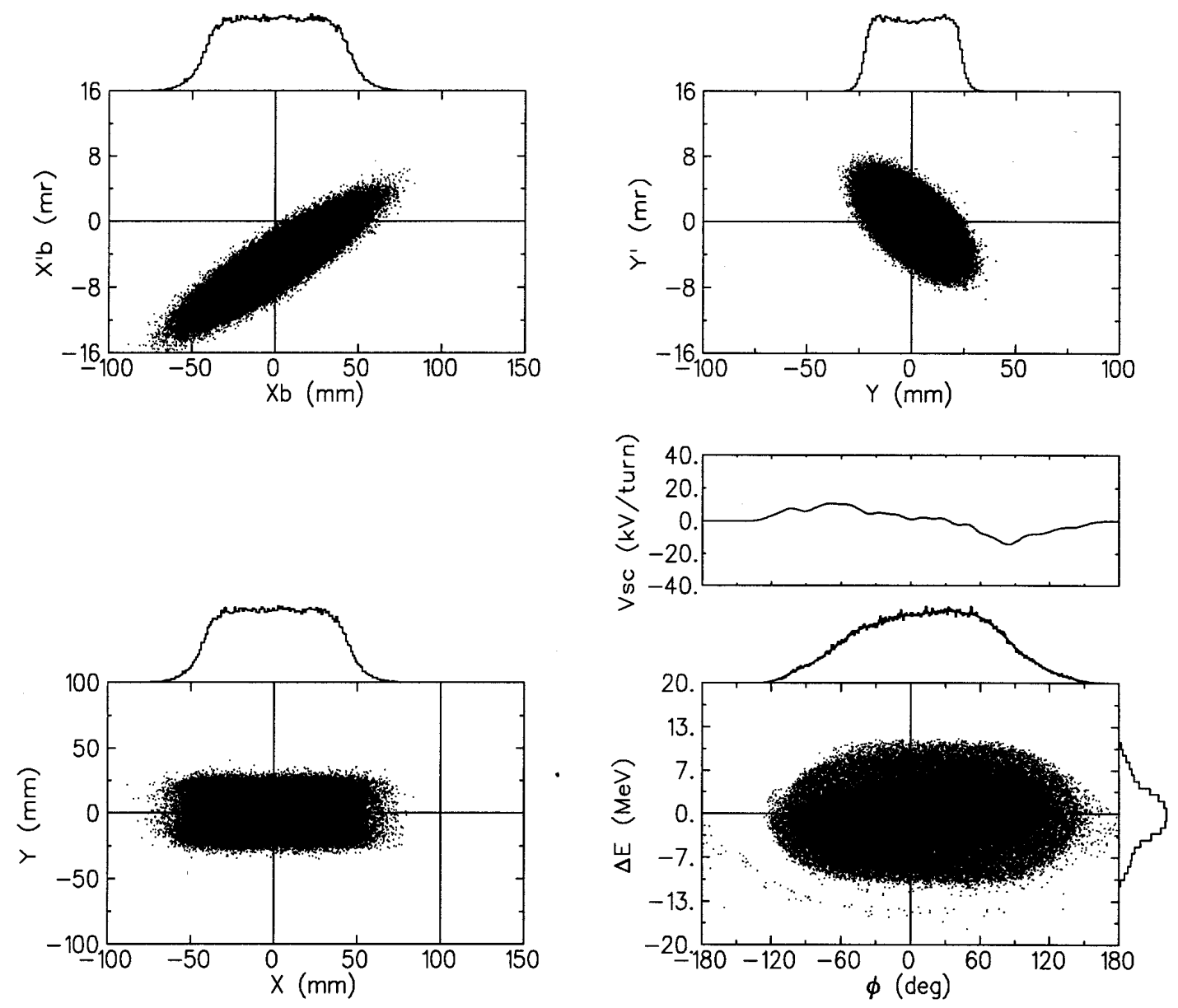

Turn \#, time (ms) $1225 \quad 1.03056$ At injection point BETAx, BETAy $15.6400 \quad 5.17342$

ETAx, ETAxp 7.74552E-02 6.67709E-03

Ts, DEO (MeV) 1000.0000 .

Vrf (kV) 40.0000

CO bump (mm) $0.927327 \quad 0.530694$

Hits: total, average 60052456612

Particles plotted, lost 97860140

Real particles in circulating beam: $2.08102 \mathrm{E}+14$

sns01_D01

Dec 21 13:06:27 1998

ACCSIM v3.5j

Figure 4: ACCSIM output for $10^{5}$ particles with size foil $100 \times 100 \mathrm{~mm}^{2}$ and thickness $2000 \mu \mathrm{g} / \mathrm{cm}^{2}$. 


\section{References}

[1] L. Landau, J. Exp. Phys. (USSR), Vol. 8, No. 4, p. 201 (1944)

[2] P. V. Vavilov, J. Exp.Teo. Phys. (USSR), Vol. 32, p. 920, (1957)

[3] U. Fano, Ann. Rev. Nucl. Sci., Vol. 13, p.1, (1963)

[4] F. W. Jones, TRI-DN-90-17, (1990)

[5]B. Schorr, CERN Program library long write-up, G110,G111, (1974)

[6] W. Borsch-Supan, J. Research Natl. Bur. Std. Vol. 65B, p.245,(1961) 